\title{
GPPS-CH-2020-0175
}

\section{AERODYNAMIC ANALYSIS OF A 7-STAGE AXIAL COMPRESSOR WITH VARIOUS TIP CLEARANCES IN ROTOR}

\author{
Wei Zhu \\ Department of Energy and Power Engineering, \\ Tsinghua University \\ zw18@mails.tsinghua.edu.cn \\ Beijing, China
}

\author{
Xue-Song $\mathrm{Li}$ \\ Department of Energy and Power Engineering, \\ Tsinghua University \\ xs-li@mail.tsinghua.edu.cn \\ Beijing, China
}

\author{
Xiao-Dong Ren \\ Department of Energy and \\ Power Engineering \\ Tsinghua University \\ rxd@mail.tsinghua.edu.cn \\ Beijing, China
}

\author{
Hong Wu \\ China United Gas Turbine \\ Technology CO., LTD \\ wuhong02@spic.com.cn \\ Beijing, China
}

\author{
Chun-wei Gu \\ China United Gas Turbine \\ Technology CO., LTD \\ gcw@mail.tsinghua.edu.cn \\ Beijing, China
}

\begin{abstract}
This paper conducts the numerical analysis on the aerodynamic performance of a 7-stage axial compressor with multi-stage computational fluid dynamic (CFD) methods. The overall performance of the axial compressor with various tip clearances in rotor, including zero clearance, small clearance, design clearance and large clearance, is analyzed under design-speed condition. The results show the decline amplitude of isentropic efficiency is different with increasing tip clearance. From the minimum clearance to small clearance, efficiency is basically unchanged. From small clearance to design clearance, efficiency decreases linearly. From design clearance to large clearance, efficiency degradation becomes larger at peak efficiency condition and smaller at near stall condition. But the efficiency of zero clearance is the lowest. Through aerodynamic analysis of various tip clearances, leakage flow increases and makes large influence on the tip flow to increase the leakage loss, leading to the decrease in efficiency with increasing tip clearance. In addition, when it is zero clearance, there is obvious corner vortex on the tip of the front stages with the scale from trailing edge to the middle chord to make large loss, especially stage 2,3,4 and 6, resulting in an obvious drop in efficiency. Thus the interaction between leakage flow and corner vortex causes different influence on the efficiency change of multi-stage compressor with various tip clearances.
\end{abstract}

\section{INTRODUCTION}

Multistage axial compressors are one of the key parts of the gas turbines and have a great influence on their efficiency and stability. The design of the compressors is focused on how to improve the load, the efficiency and the surge margin in the modern heavy-duty gas turbines. Consequently, the internal turbulent flow of the multistage highly loaded compressor becomes more complex to make more challenging aerodynamic designs. The effects of the tip leakage flow, the rotor-stator interaction, the interstage matching and the shock wave should be carefully studied (Denton,1993). Among these complex flow phenomena, it is necessary to pay more attention to the analysis of tip leakage flow. Due to the tip clearance between the rotor blade and the upper endwall, the leakage flow from the pressure surface to the suction surface is produced by the action of the pressure difference between the suction surface and the pressure surface. In addition, the leakage flow interacts with the main flow and the shock wave to produce the main leakage vortex and the secondary leakage vortex respectively, resulting in negative impact on the performance and stability of the compressors (Du et al., 2013; Bergner et al., 2006; Hofmann , 2003).

With the rapid development of computational fluid dynamic (CFD) technology, the multistage CFD method is widely applied for the aerodynamic analysis and optimization of modern compressors (Wellborn,2001; Brilliant et al., 2004; Belamri et al., 2005a; Belamri et al., 2005b; Mansour et al., 2008). Many researchers have carried out 3-D CFD numerical studies on the aerodynamic performances with various tip clearances in rotor. Wang et al (Wang et al., 2009) took the seventh and eighth stages of a multi- 
stage subsonic axial flow compressor as the research object to obtain the optimal compressor performance when it is zero clearance. Li et al. (Li et al., 2008) used the rotor blade of the twelfth stage of a high pressure compressor to find there was the best tip clearance value, but not the zero clearance. Teng et al. (Teng et al., 2018) made research on a five-stage axial compressor to compare with the overall performance of the minimum and the maximum tip clearance to present the efficiency and pressure ratio decreased slightly with the increasing tip clearance. Syed et al. (Syed et al., 2016) investigated the front 1.5 stage of a 3-stage compressor to obtain the optimal tip clearance by comprehensive comparison of efficiency and operating range. Therefore, when the compressor research objects are different, the aerodynamic performance caused by various tip clearances is also different.

Meanwhile, the flow loss and instability characteristics of compressor tip clearance are also analyzed widely and carefully. Denton (Denton,1993) pointed out that nearly $1 / 3$ of the rotor blade loss occurred in the tip area when the sources and influences of various losses of turbine machinery were analyzed. Currently, the research on tip leakage flow includes two types of compressors: subsonic and transonic, which focuses on the unsteady characteristics of the leakage flow, such as the structure of the leakage flow under different working conditions and the relationship between the leakage flow and spike-type stall precursor. For the low-speed E3 Rotor, when operating point approaches to the stall point, the interface between tip leakage vortex and the main flow gradually moves to the upstream passage of rotor blade with the decrease in the flow coefficient (Hoying,et al., 1999); And a criterion for spike-type stall precursor is presented, that is, the tip leakage flow needs to be satisfied at the same time when it occurs reflux near the trailing edge and overflow near the leading edge (Vo et al., 2008). For the above criterion, Yamada et al. (Yamada et al., 2011; Yamada et al., 2017) used the multistage centrifugal compressor and axial compressor to analyze tip stall process and detailed internal flow to discover that the formation of spike-type stall precursor was directly related to tip leakage vortex, which formed a large vortex in the passage, thus blocking tip passage and causing the overflow of leading edge. In addition, with the increase in compressor stage load, the research on tip leakage flow in transonic compressor is increasing. Although it is similar in the basic structure and mechanism of tip leakage vortex between subsonic and transonic, compressor, the research on transonic compressor also pay more attention on the mutual interference mechanism between leakage vortex and shock wave due to the higher pressure gradient and the exist of shock wave. Hoffman (Hofmann, 2002) found there was a large tip loss in multistage transonic axial compressor, which was manifested in the interaction between shock wave and tip leakage flow, resulting in sudden deceleration of flow and changing the distribution of leakage vortex to form tip blocking area. And many scholars considered that the leakage vortex may break under the near-stall condition and produced more low-energy fluid areas at the blade tip passage (Yamada, et al., 2011; Schlechtriem, 1997; Shi, 2013). In terms of experimental measurement, Brandstetter (Brandstetter, 2018) presented that the fragmentation of tip leakage vortex led to the disturbance of a large range of secondary flow structures through PIV measurement technology.

Therefore, tip leakage flows in subsonic/transonic compressors are more than complicated, especially in the transonic compressors with larger adverse pressure gradient and stronger unsteady effect. The changes of tip clearance have different effects on the aerodynamic performances of different compressor types. Therefore, this paper adopts the multistage CFD methods to analyze the influence mechanism of the various tip clearances on the aerodynamic performance of a 7-stage axial compressor, which is sketched in Figure1.

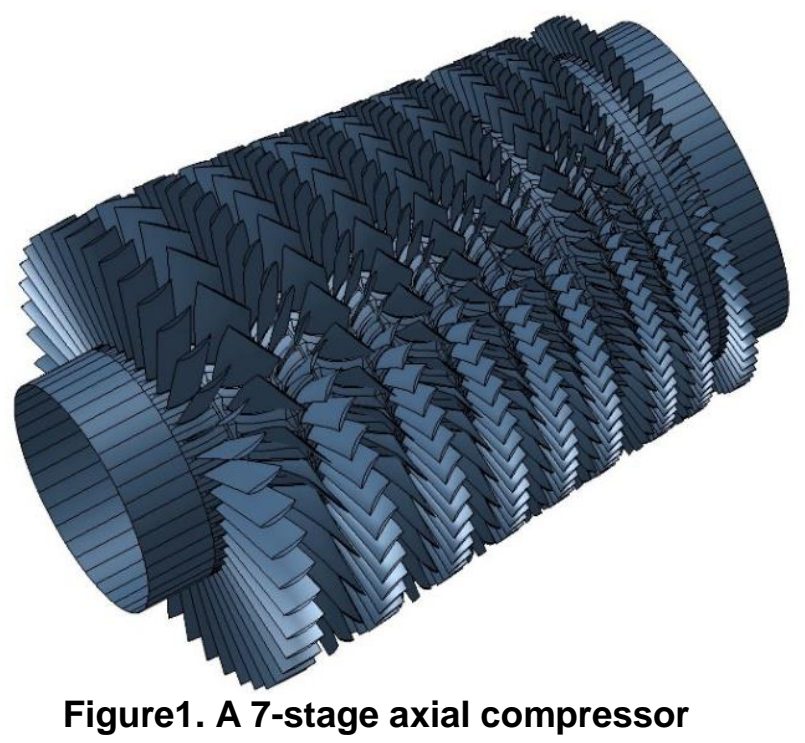

\section{NUMERICAL SIMULATION METHOD}

In this paper, the single-passage steady numerical simulation is carried out for the 7-stage axial compressor at the design rotational speed. The compressor has 16 blade rows, including the inlet guide vane (IGV), seven stages (three transonic stages and four subsonic stages), and the outlet guide vane (OGV). The design tip clearances of each rotor considered to be simple constant 
span gaps and the value is $0.5 \mathrm{~mm}$. The relative tip clearance (relative to chord) of the first rotor and last rotor is $0.7 \%$ and $1.67 \%$, respectively. The qualitative indication with various tip clearances is also given, including zero clearance, small clearance, design clearance and large clearance, as shown in Figure 2. And other detailed compressor design specifications cannot be provided in this paper because of the proprietary nature of the information.
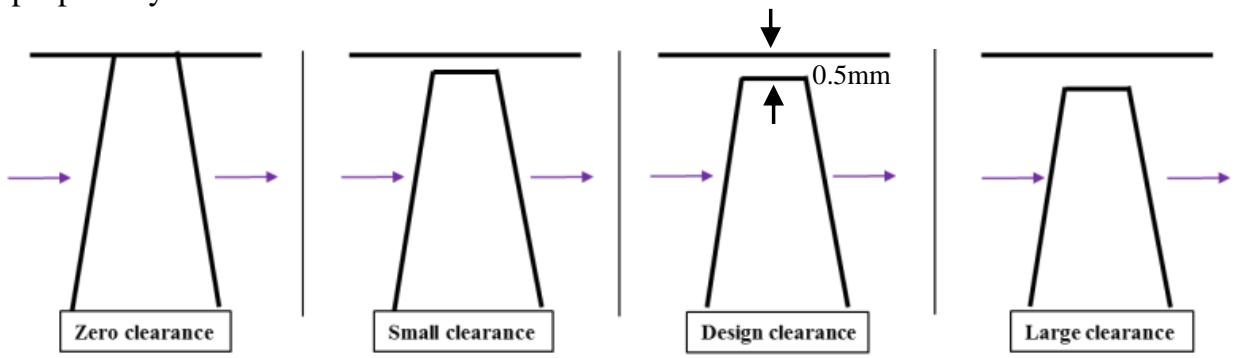

Figure 2. Qualitative indication with various tip clearances

Multistage CFD methods are adopted in the aerodynamic analysis with the commercial CFD software "Fine/Turbo", where the 3-D Reynolds-Averaged N-S equations are solved. The Spalart-Allmaras(S-A) turbulence model (Spalart, 1994) and the central difference scheme for spatial discretization are employed for the solver. Moreover, a multi-grid approach is applied to accelerate the convergence. The CFL number is 3 . In addition, the mixing plane approach with Full Non Matching Technique is used as the rotor-stator interface strategy. In the boundary condition setting, total temperature, total pressure and axial inlet conditions are given at the inlet, while a radial pressure gradient to match simple radial equilibrium law is specified at the outlet. Moreover, the periodic conditions is applied on the pitchwise boundaries of the flow domain. Nonslip and adiabatic conditions are adopted for all solid walls. The mesh is generated with the commercial CFD software "Auto-Grid", using a HOH (inlet, O, and outlet blocks) mesh topology. The rotor tip clearances are filled with butterfly mesh blocks, as demonstrated in Figure 3. For the convenience of direct comparison, the same mesh division is given for various tip clearances conditions.

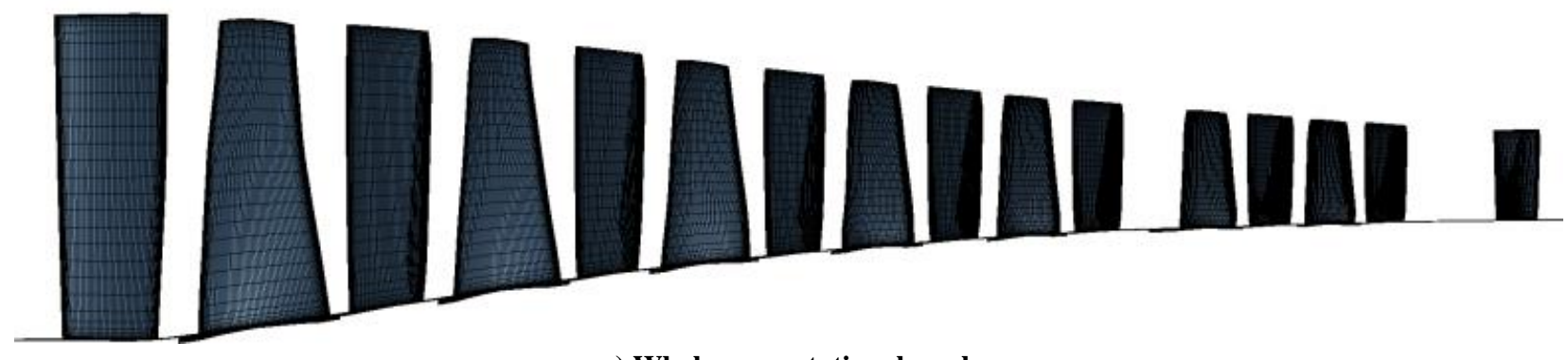

a) Whole computational mesh

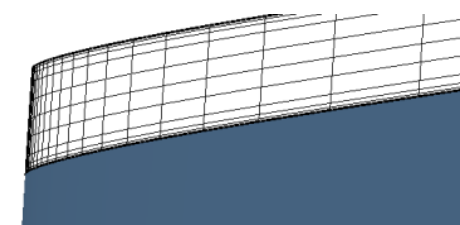

b) Mesh at the rotor tip

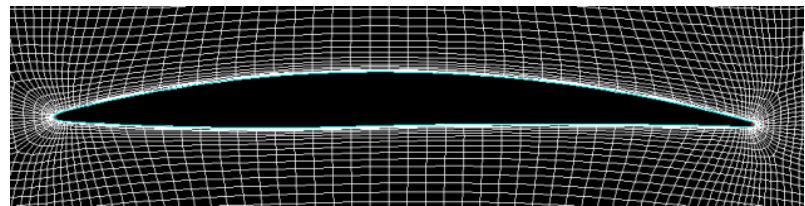

c) B2B Mesh at 0.5span

Figure 3. Computational domain and mesh

The mesh independence validation is conducted to make sure the computational results independent of grid distribution. Considering the satisfy of $\mathrm{y}+$ and the cost of CFD calculation, equal proportion encryption (all $\mathrm{i}, \mathrm{j}, \mathrm{k}$ resolutions proportionally increased ) is used on the basis of single channel reference grid 8.84 million to obtain 13.9 million for direct comparison. $\mathrm{y}+$ is less than 3. The overall performance of near stall point (NS), design point (DP) and near-choke point (NC) is compared, and the characteristic curves of isentropic efficiency-relative mass flow rate and relative pressure ratio - relative mass flow rate are shown in Figure 4. The results show the isentropic efficiency, the relative pressure ratio and the relative mass flow rate (dimensionless by DP) at the corresponding point are basically consistent with the grid encryption. Moreover, 8.84 million grids can also obtain the change trend of overall performance. Then a total mesh size of 8.84 million for the 16 blade passage is selected for subsequent calculation and analysis. 


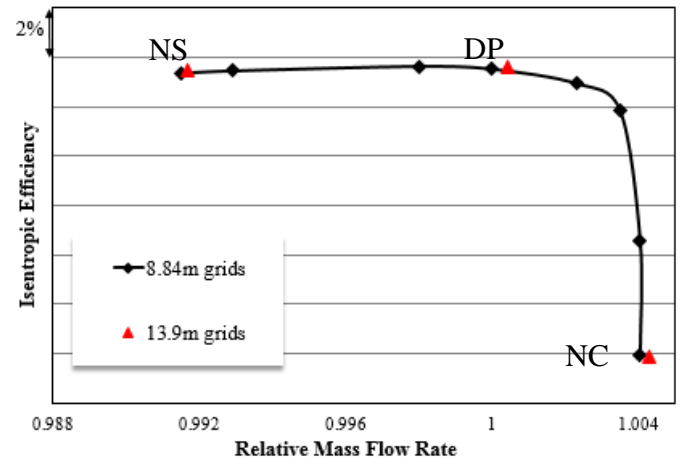

a) Isentropic Efficiency- Relative Mass Flow Rate

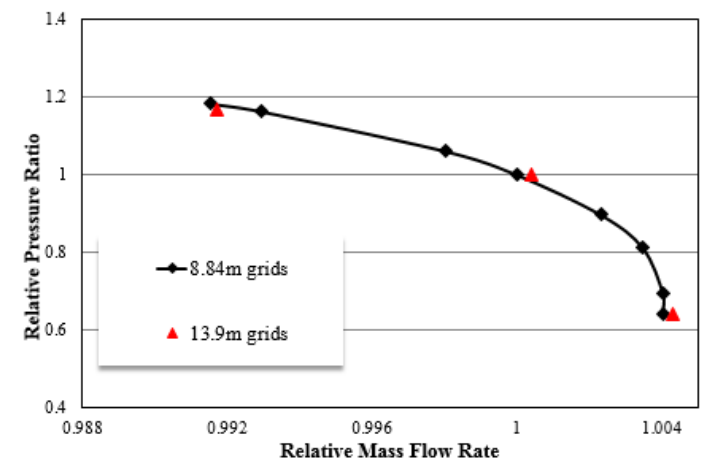

b) Relative Pressure Ratio- Relative Mass Flow Rate

Figure 4. Mesh independence validation

\section{ANALYSIS OF OVERALL PERFORMANCE}

The characteristic curves of isentropic efficiency-relative mass flow rate and relative pressure ratio - relative mass flow rate under various relative tip clearance (dimensionless by design tip clearance) are calculated, as shown in Figure 5. And the curves of isentropic efficiency- relative pressure ratio are also shown in Figure 5. The results show the isentropic efficiency of each relative tip clearance (RTC) has the same changing trend, and there exist the peak efficiency points (PE). The changing trend of the relative pressure ratio is also the same, which increases gradually with the decrease in the relative mass flow. Compared with various tip clearances, the isentropic efficiency, the relative pressure ratio and the max relative mass flow increase with the decrease in tip clearance. However, the overall performance is basically the same between RTC0.06 and RTC0.2. Meanwhile, RTC0 is taken as a reference condition, the isentropic efficiency, relative pressure ratio and the max relative mass flow are the minimum. In addition, at the condition of RTC1 (design tip clearance), the operating range is the highest as compared to all tip clearances.

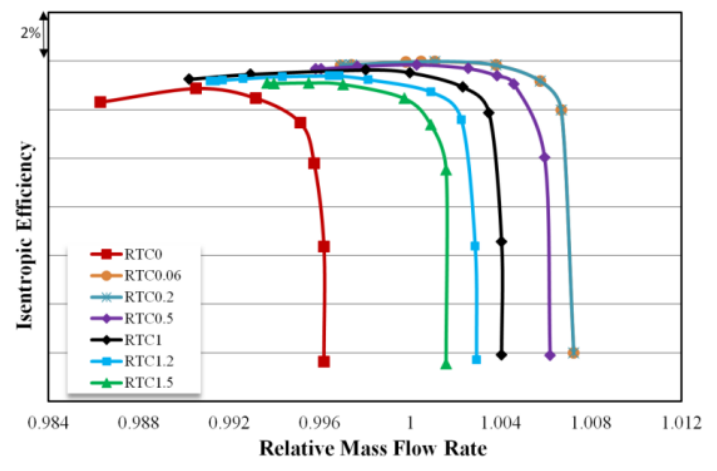

a) Isentropic Efficiency- Relative Mass Flow Rate

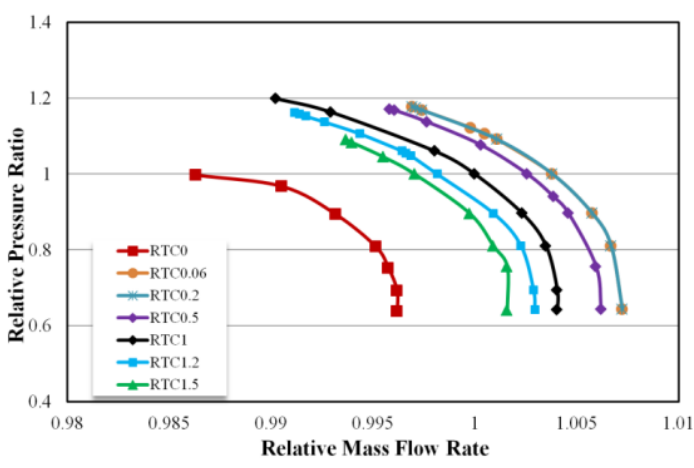

b) Relative Pressure Ratio- Relative Mass Flow Rate

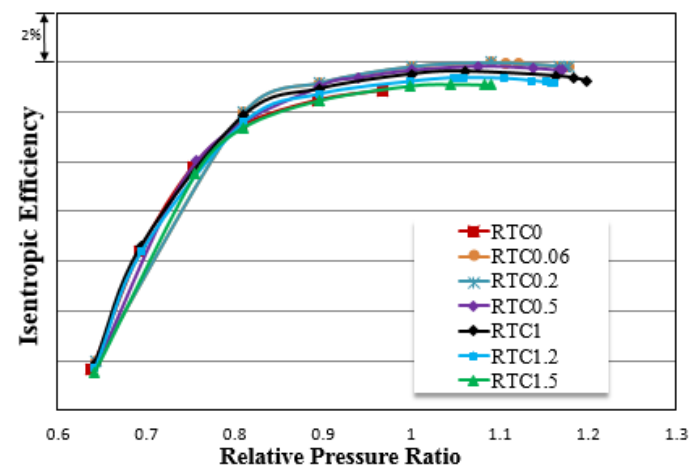

c) Isentropic Efficiency - Relative Pressure Ratio

Figure 5. Compressor characteristic curves with various tip clearances

\section{INFLUENCE OF VARIOUS TIP CLEARANCES ON COMPRESSOR PERFORMANCE}

According to previous analysis of the overall performance, the influence of various tip clearances on compressor performance parameters is obvious and regular. Therefore, the peak efficiency points (PE) and near stall (NS) points are selected to analyze the variation of isentropic efficiency and relative pressure ratio with different tip clearance values, as shown in Figure 6 and Figure 7 
respectively. Figure 6(a) shows the efficiency performance decreases gradually with the increase in tip clearance. But the decline amplitude is different and can be divided into three sections. From RTC0.06 to RTC0.2, efficiency is basically unchanged. From RTC0.2 to RTC1, efficiency decreases linearly. From RTC1 to RTC1.5, efficiency degradation becomes larger. Figure 6(b) shows the relative pressure ratio performance decreases gradually with the increase in tip clearance. But the isentropic efficiency and relative pressure ratio of RTC0 are the lowest.

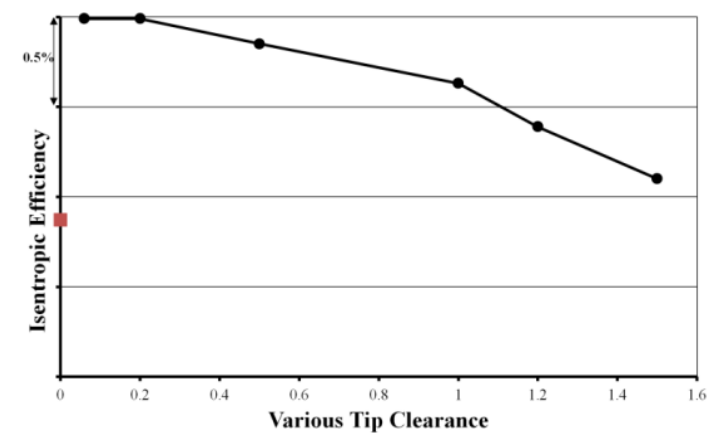

a) Isentropic Efficiency- Various Tip Clearance

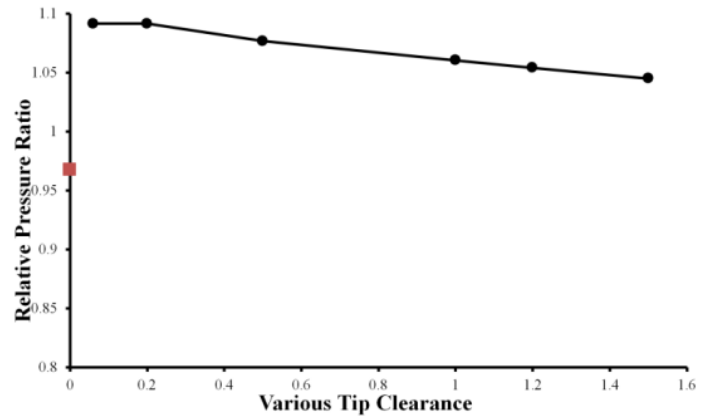

b) Relative Pressure Ratio- Various Tip Clearance

\section{Figure 6. Tip clearance influence on the full-stage compressor performance at the PE conditions}

Figure 7(a) also show the efficiency performance decreases gradually with the increase in tip clearance. And the decline amplitude is also different and can be divided into three sections. From RTC0.06 to RTC0.2, efficiency is basically unchanged. From RTC0.2 to RTC1, efficiency decreases linearly and efficiency degradation is the largest. From RTC1 to RTC1.5, efficiency decreases linearly with lower slope. Figure 7(b) shows the relative pressure ratio performance varies differently with the increase in tip clearance. From RTC0.06 to RTC0.5, the relative pressure ratio changes little. RTC1 has the largest pressure ratio, then decreases with the increase in tip clearance. But the isentropic efficiency and relative pressure ratio of RTC0 are the lowest. In general, the change of overall performance with various tip clearances is different from the previous research results done by many scholars. Therefore, it is necessary to conduct in-depth aerodynamic analysis to explain the flow mechanism.

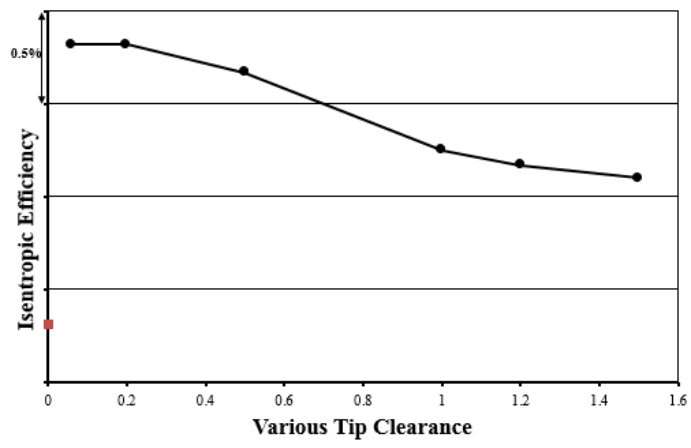

a) Isentropic Efficiency- Various Tip Clearance

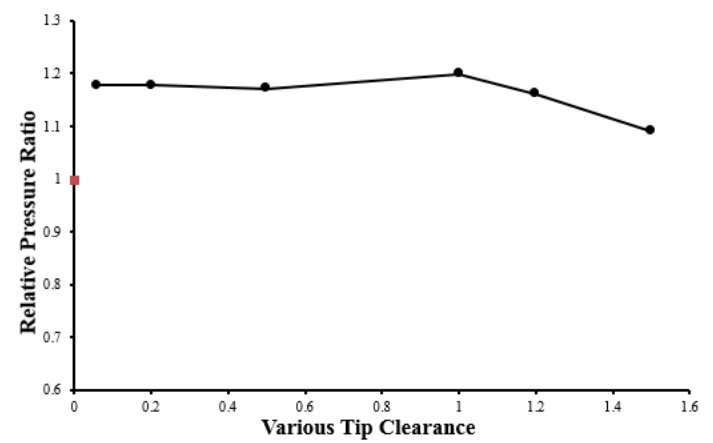

b) Relative Pressure Ratio- Various Tip Clearance

Figure 7. Tip clearance influence on the full-stage compressor performance at the NS conditions

\section{ANALYSIS OF TIP LEAKAGE FLOW WITH THREE TIP CLEARANCES}

The influence mechanism of various tip clearances on compressor performance is first analyzed based on the flow field with three tip clearances, including RTC0.2, RTC1 and RTC1.5. Meanwhile, R3 as one of three transonic rotors and R4 as one of four subsonic rotors are selected. The relative Mach number distribution at $98 \%$ span of R3 and R4 are shown in Figure 8. It can be seen the complex flow like shock wave and tip leakage flow both appear, and the interaction between shock wave and leakage flow causes the internal turbulent flow with higher unsteady and nonlinearity. Compared with different tip clearances, the relative Mach number changes significantly at $98 \%$ span with the increase in tip clearance. The location of shock wave is basically the same, but the strength changes a lot. As shown in Figure 8(a), the strength of shock wave of R3 gradually weakens and the leakage flow increases with the increase in tip clearance, resulting in larger low-speed zone and more obvious blocking effect on the main passage to cause the decrease in efficiency. In addition, although the relative Mach number of R4 is smaller than R3 and the shock wave phenomenon is not obvious, the flow rule is similar to R3. 

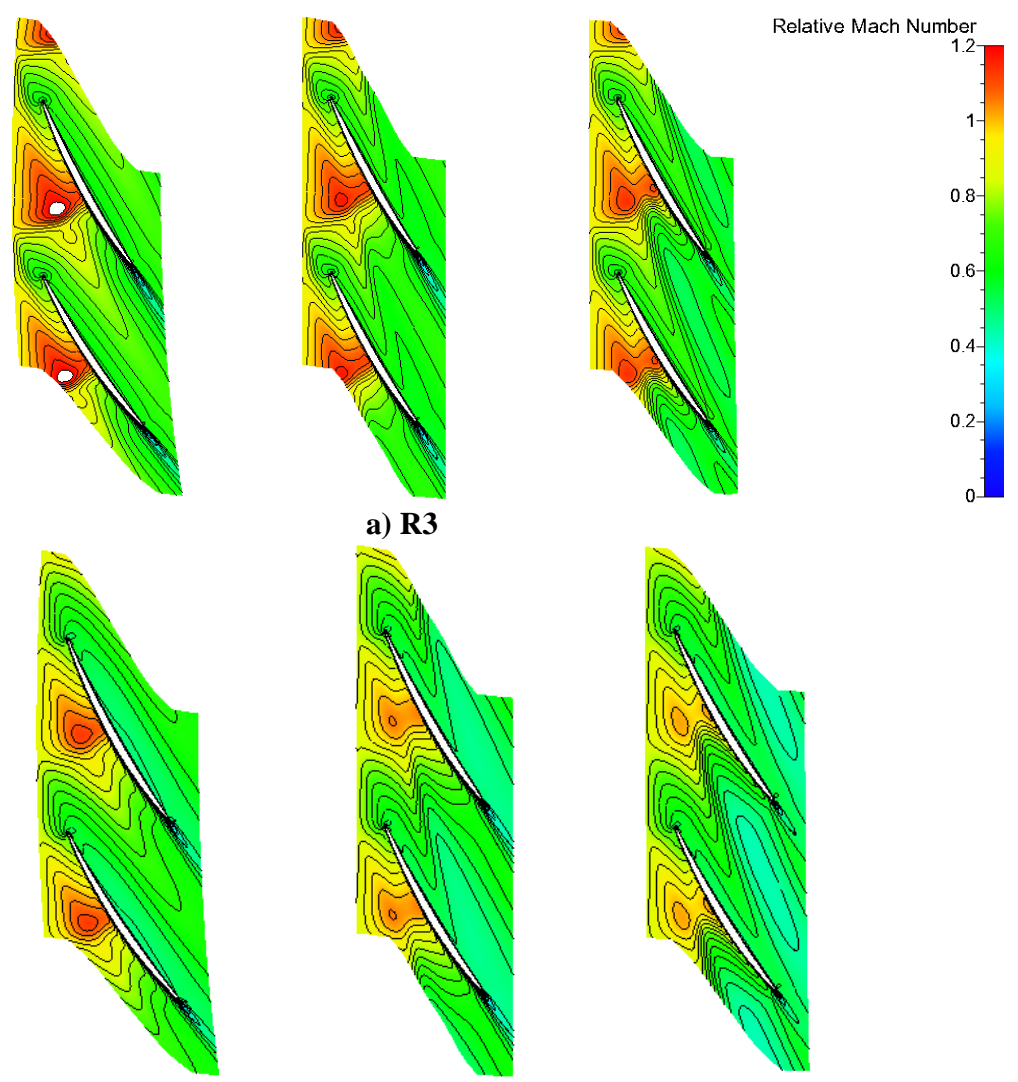

b) $\mathbf{R} 4$

Figure 8. Relative Mach contour of RTC0.2 (Left), RTC1 (Middle), RTC1.5 (Right) at 98\% span

The static pressure distribution of R3 and R4 at 98\% span is further analyzed, as shown in Figure 9. In Figure 9(a), it is found that the change of tip clearances has obvious influence on the static pressure distribution of R3, with some certain influence on the front half of the pressure surface and the rear half of suction surface. With the increase of tip clearances, the pressure and pressure gradient decrease gradually, which further explains the influence of leakage flow on shock wave, and then changes the pressure distribution of blade tip. Similarly, Figure 9(b) shows the static pressure distribution of R4 also change with the increase of tip clearances but more obvious than R3, no matter on the pressure surface or suction surface. Therefore, tip leakage flow has more influence on R4 than R3.

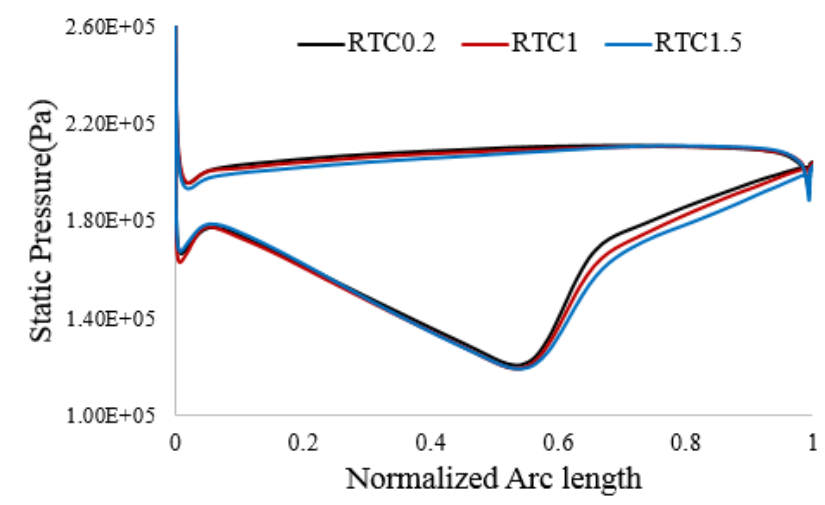

a) $\mathbf{R 3}$

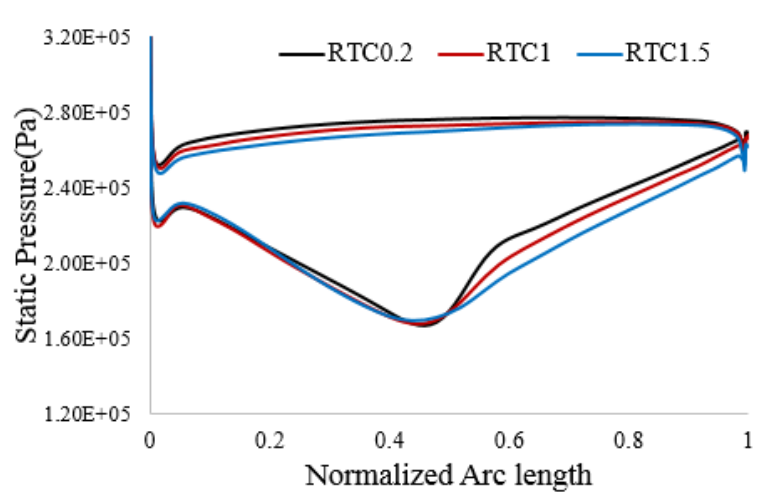

b) $\mathbf{R 4}$

Figure 9. Static Pressure distribution at $98 \%$ span

\section{ANALYSIS OF CORNER VORTEX WITH/WITHOUT TIP CLEARANCE}

Furthermore, the aerodynamic analysis with/without tip clearance is analyzed in detail with RTC0 and RTC1. Figure 10 demonstrates the relative Mach number distribution of each blade of RTC0 and RTC1 at 98\%span. In Figure 10(a), there is obvious local low-speed zone on the tip of the front stages with the scale from trailing edge to the middle chord to form large corner vortex, especially stage 2, 3, 4 and 6, which is produced by the interaction between the boundary layer on the blade wall and the boundary layer on the upper endwall. Meanwhile, there is obvious shock wave around the leading edge of the rotor of the first stage and a 
large low-speed zone occupies most area of the blade passage to block the main flow. No shock wave occurs in other stages due to the low Mach number. However, the circumferential secondary flow is obvious because of the pressure difference between the adjacent pressure surface and suction surface.

In general, with RTC0 the flow field at the tip of the compressor is very complex. These complex flows lead to a number of flow losses in the compressor, including shock wave loss, corner vortex blockage loss and secondary flow loss, which cause the reduction of the overall performance of the compressor.

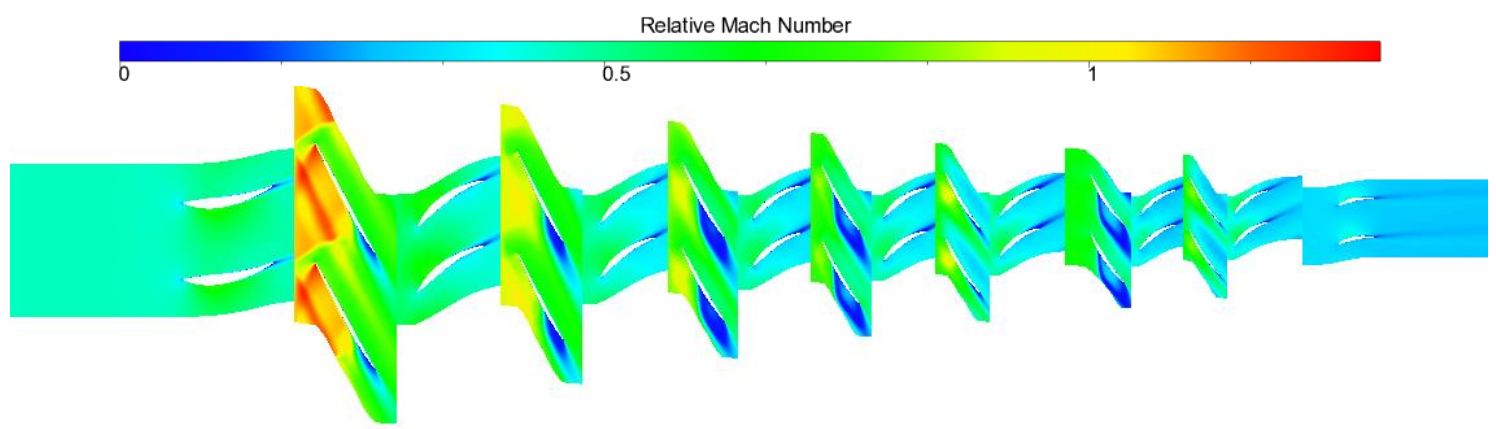

a) RTC0

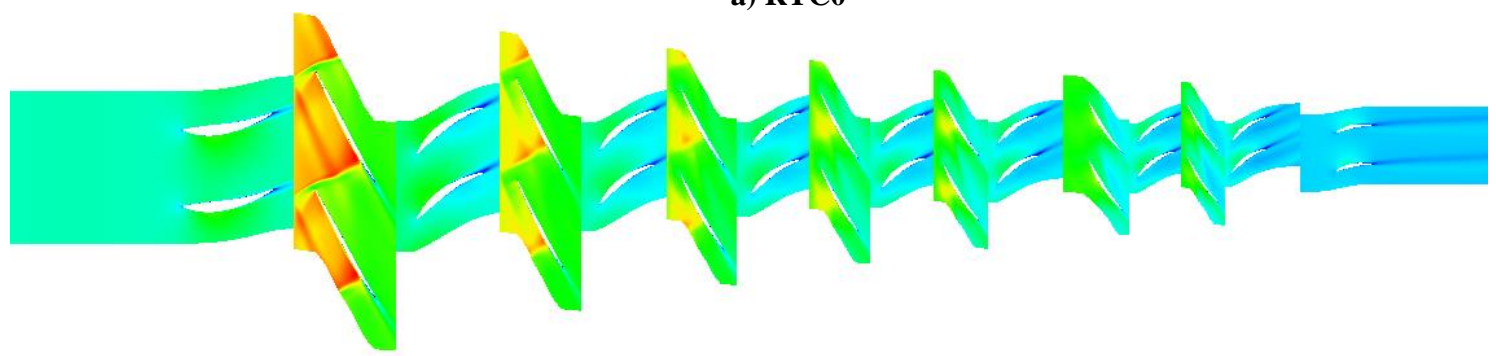

b) RTC1

Figure 10. Relative Mach contour at $98 \%$ span

In Figure 10(b), there is no corner vortex on the suction surface of rotor blade. The leading edges of the front three stages all present an obvious detached shock wave, whose upper half is a bow shock wave and the lower half is a positive shock wave near the vertical suction surface of the adjacent blade. In addition, the leakage flow interacts with the main flow and the shock wave to produce leakage vortex. A large low speed zone which occupies most area of the blade passage to block the main flow. No shock wave occurs in the last four stages due to the low Mach number, but the leakage flow and the circumferential secondary flow are obvious in the blade passage.

In general, with RTC1 the flow field at the tip of the compressor is also very complex. Compared with RTC0, there is no corner vortex and the flow field is more regular. However, these complex flows lead to a number of flow losses in the compressor, including shock wave loss, leakage loss and secondary flow loss, which cause the reduction of the overall performance of the compressor.

Above all, corner vortex and leakage flow play a dominant role in the loss performance with/without clearance in the tip region, respectively. It is necessary to analyze the flow field along the radial direction. Figure 11 shows the limiting streamlines on the suction side of each rotor blade with/without tip clearance. In Figure 11(a), with RTC0 there is obvious large corner vortex on the tip of the front stages. With the increase in stage number (except stage 5 and stage 7 ), the tip area covered by the corner vortex increases and it basically occupies the flow passage from the trailing edge to the middle chord, which is consistent with Figure 10(a). In Figure 11(b), with RTC1 there is no corner vortex on the suction side of each rotor blade and no obvious flow separation, which is consistent with Figure 10(b).
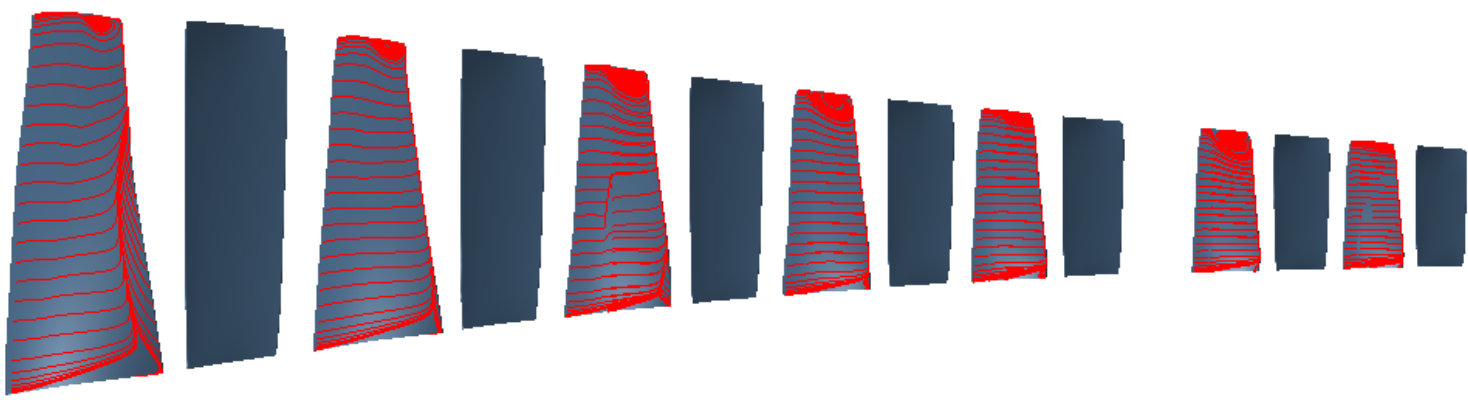

a) RTC0 


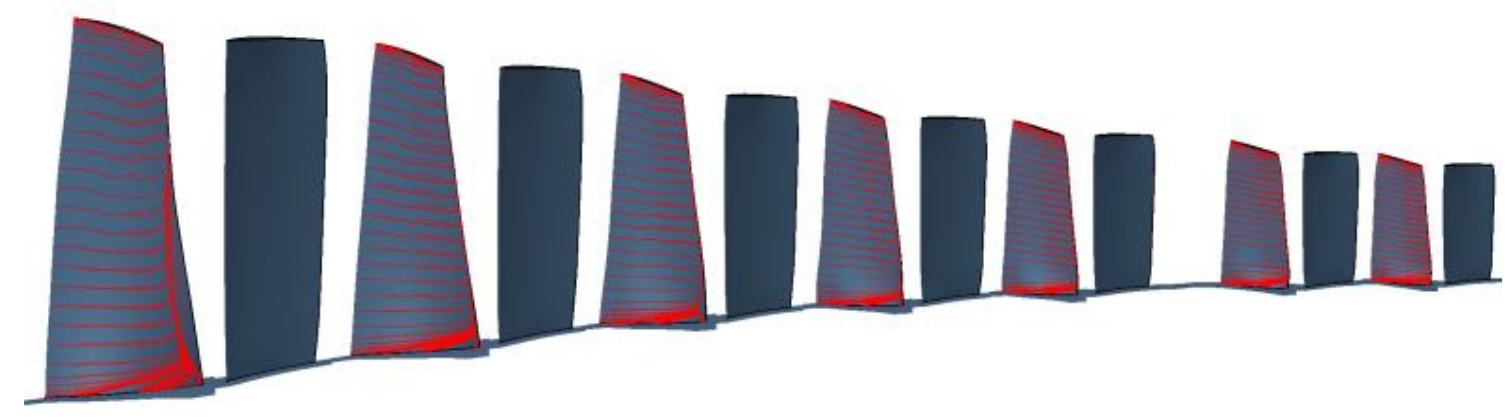

b) RTC1

Figure 11. Limiting streamlines on the suction side of each rotor blade

\section{ANALYSIS OF STAGE PERFORMANCE WITH/WITHOUT TIP CLEARANCE}

In order to deeply analyze the effect of tip corner vortex on the change of aerodynamic performance, Figure 12 shows the stage overall performance variation with/without tip clearance at the same overall pressure ratio conditions. In Figure 12(a), the variation trend and amplitude of each stage efficiency are different, and the adjacent two stages affect each other. By comparing the stage efficiency results with/without tip clearance, the stage efficiency of RTC1 is higher than that of RTC0 at rear 6 stages, except the first stage. Moreover, the efficiency difference of each stage is different. From the second stage to the fourth stage, the efficiency difference gradually becomes larger. The efficiency of stage 5 and stage 7 is almost same and the efficiency of stage 6 has the largest difference, which is consistent with the results in Figure 10(a) and Figure 11(a). In Figure 12(b), the variation trend and amplitude of each stage pressure ratio are also different. Except stage 6, the pressure ratio of other stages of RTC0 is larger than RTC1.

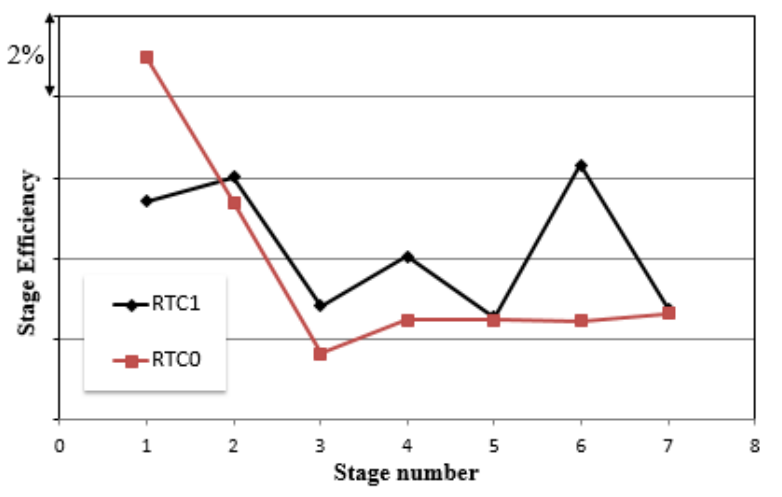

a) Stage Efficiency

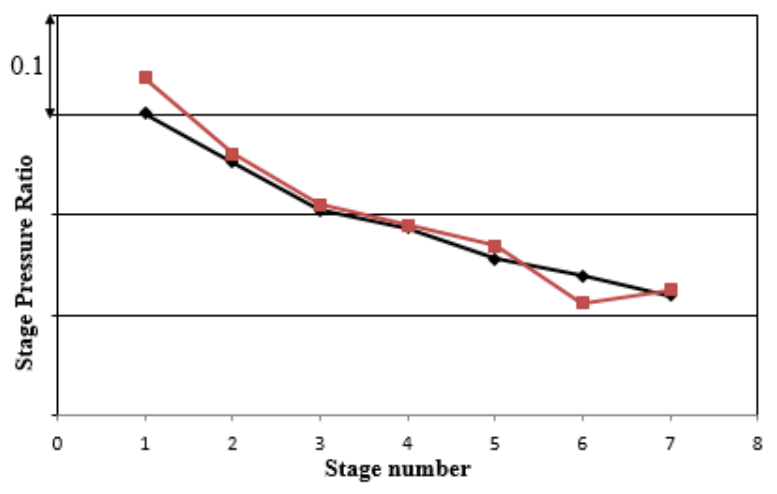

b) Stage Pressure Ratio

Figure 12. Stage performance with/ without tip clearance

The radial distribution of stage efficiency at all stages is given below, as shown in Figure 13. Figure 13(a) demonstrates the first stage efficiency of RTC0 is higher than that of RTC1 from blade root to 0.8span, and the result of tip region is basically the same. It is well known that RTC1 has a large loss caused by tip clearance leakage flow which mainly reduce shock wave intensity to make the decrease of boost capacity. And the decrease in efficiency due to the leakage flow losses could be of the same order of magnitude with corner vortex blockage loss of RTC0. From Figure 13(b) to 13(d), the stage efficiency of RTC0 is lower than that of RTC1 from 0.6span, 0,7span and 0.8span to the tip respectively, indicating that the area of local corner vortex of RTC0 becomes larger, causing larger loss than that of RTC1 due to leakage flow. In Figure 13(e) and 13(g), RTC0 has a higher efficiency of stage 5 and stage 7 than RTC1 from 0.6 span and 0.8span to the tip respectively, because very small corner vortex occurs in RTC0, while tip leakage flow loss is still large in RTC1. Figure 13(f) shows the sixth stage efficiency of RTC0 is much lower than that of RTC1 from 0.5 span to the tip, indicating that the area of local corner vortex of RTC 0 is further increased than the front four stages, causing a larger loss. Therefore, the loss difference between corner vortex and leakage flow determines the different influence on stage performance with/without tip clearance. 


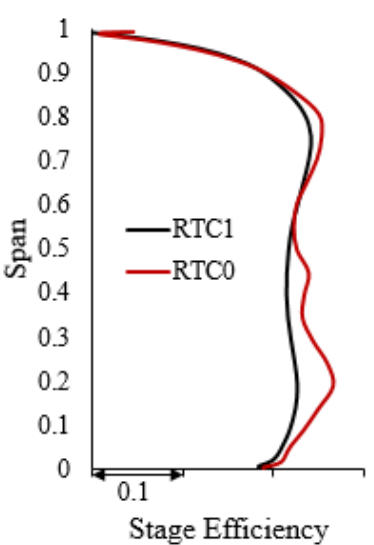

a) Stage 1

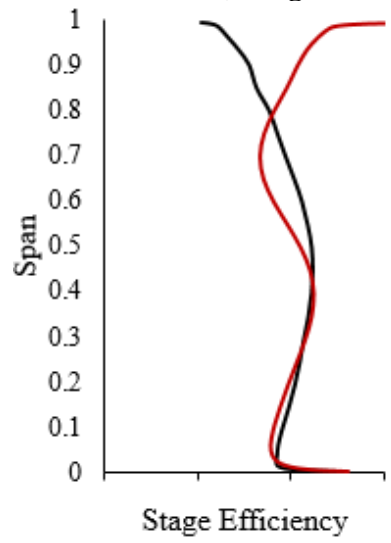

e) Stage 5

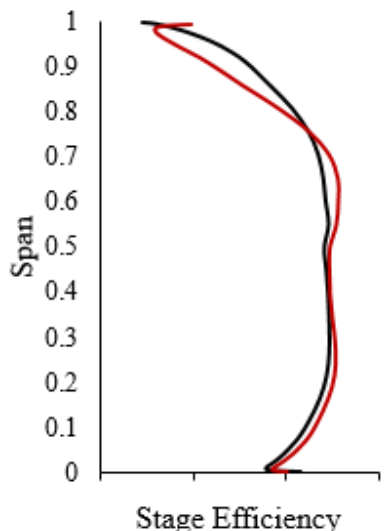

b) Stage 2

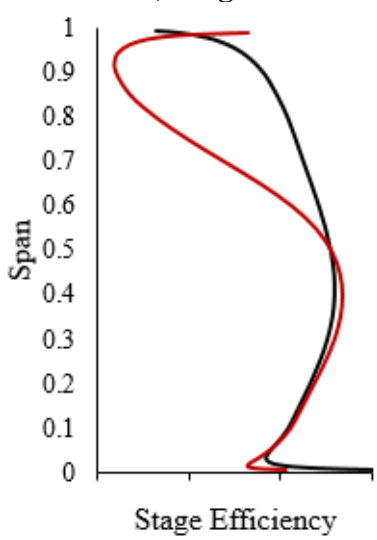

f) Stage 6

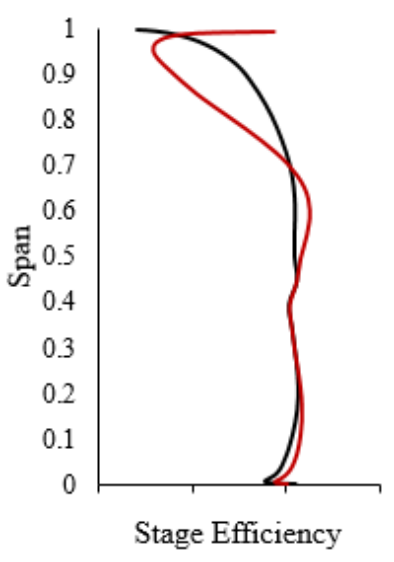

c) Stage 3

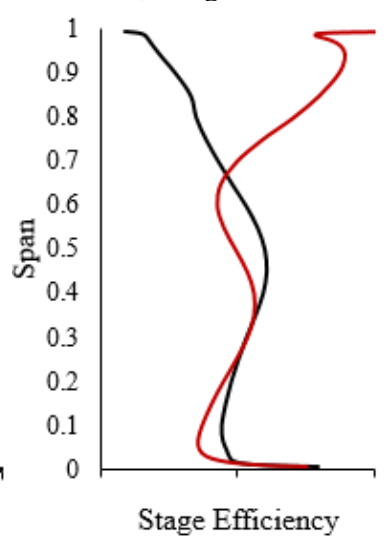

g) Stage 7

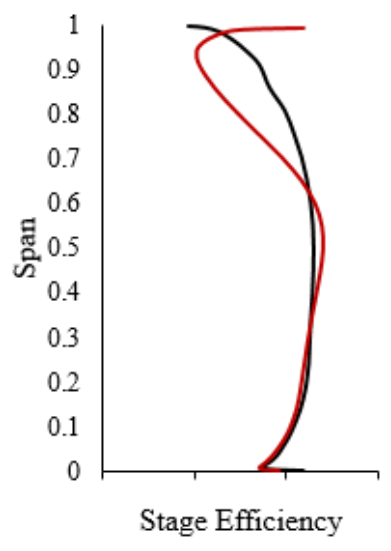

d) Stage 4

Figure 13. Radial distribution of Stage Efficiency with/ without tip clearance

There is still some limitation of this paper. Currently, the first 3-stage axial compressor test has been carried out and CFD method is verified. But the 7-stage axial compressor test is not conducted because that the test facilities are still under construction. After that, some measurements of the velocities and the pressures will be conducted. Then the test results will be presented in the future research paper.

\section{CONCLUSIONS}

This paper conducts the numerical analysis on the aerodynamic performance of a 7-stage axial compressor to analyze the overall performance and the detailed flow field with various tip clearances in rotor. The results show the decline amplitude of isentropic efficiency is different with increasing tip clearance. From the minimum clearance to small clearance, efficiency is basically unchanged. From small clearance to design clearance, efficiency decreases linearly. From design clearance to large clearance, efficiency degradation becomes larger at peak efficiency condition and smaller at near stall condition. But the efficiency of zero clearance is the lowest.

Furthermore, the larger the tip clearance, the stronger the leakage flow, which plays a dominant role to cause the leakage loss and the obvious decrease of efficiency. Compared with the flow field and stage performance with/without tip clearance, the results show large tip corner vortex exists in the front stages when it is without tip clearance, which results in the increase of the internal loss of the full-stage compressor and the overall performance is lower than that with tip clearance. In addition, the corner vortex area becomes larger with the increase in stage number (except stage 5 and stage 7), which leads to the increase of the internal loss of single stage.

It can be concluded that the interaction between corner vortex and leakage flow leads to the different influence of various tip clearances on compressor performance. When it is zero clearance, there is no leakage flow but the corner vortex decreases efficiency. When it is design clearance, there is no corner vortex but the leakage flow decreases efficiency. Therefore, when it is a small clearance, the leakage flow and corner vortex may restrain each other to some extent, so that the efficiency can be improved.

\section{Nomenclature}

CFD computational fluid dynamic

IGV inlet guide vane 


$\begin{array}{lc}\text { OGV } & \text { outlet guide vane } \\ \text { DP } & \text { design point } \\ \text { NC } & \text { near chock } \\ \text { NS } & \text { near stall } \\ \text { RTC } & \text { relative tip clearance } \\ \text { PE } & \text { peak efficiency }\end{array}$

\section{ACKNOWLEDGMENTS}

This work is supported by Project 2017-II-0007-0021 of the National Science and Technology Major Project of China.

\section{References}

Denton, J. D. (1993). Loss mechanisms in turbomachines. Journal of Turbomachinery, vol. 115, pp. 621-656.

$\mathrm{Du}$ J, et al. (2013). Flow structures in the tip region for a transonic compressor rotor. Journal of Turbomachinery, $135(3)$ : 031012. Bergner J, et al. (2006). Short length-scale rotating stall inception in a transonic axial compressor: Experimental investigation. Volume 6: Turbomachinery, Parts A and B, pp :131-140.

Hofmann W H, Ballmann J.(2003). Some aspects of tip vortex behavior in a transonic turbocompressor. ISABE, pp, 1223:2003.

Wellborn S R, Delaney R A. (2001). Redesign of 12-Stage Axial Flow Compressor Using Multistage CFD. ASME, GT2001-0351

Brilliant L, et al. (2004). Application of Multistage CFD Analysis to Low Compressor. Design. ASME, GT2004-54263.

Belamri T, et al. (2005). CFD analysis of a 15 Stage Axial Compressor Part 1: Methods. ASME, GT2005-68261.

Belamri T, et al. (2005). CFD analysis of a 15 Stage Axial Compressor Part 2: Reaults. ASME, GT2005-68262.

Mansour M L, et al. (2008,). Validation of Steady Average-Passage and Mixing Plane CFD Approaches for the Performance Prediction of a Modern Gas Turbine Multistage Axial Compressor. ASME, GT2008-50653.

Wang X F, et al. (2009). Numerical simulation study on multistage compressor performance with changing tip clearance. Science Technology and Engineering, 9(12): 3483-3486.

Li Q, et al. (2008). Numerical simulation of high pressure axial compressor with tip clearance in rotor. Computation Simulation, 25(11): 44-47

Teng X, et al. (2018). The influence of geometry deformation on a multistage compressor. ASME.

Syed N D, et al. (2016). Effect of tip clearance and rotor-stator axial gap on the efficiency of a multistage compressor. Applied Thermal Engineering, pp, 988-995.

Hoying D A,et al. (1999). Role of blade passage structures in axial compressor rotating stall inception. Journal of Turbomachinery, 121(4): 735.

Vo H D, et al. (2008). Criteria for spke initiates rotating stall. Journal of turbomachinery, 130(1): 011023.

Yamada K, et al. (2011).The Role of Tip Leakage Vortex Breakdown in Flow Fields and Aerodynamic Characteristics of Transonic Centrifugal. ASME.

Yamada K, et al. (2017).Large-Scale Detached-Eddy Simulation Analysis of Stall Inception Process in a Multistage Axial Flow Compressor. ASME.

Hofmann W and Ballmann J. (2002). Tip clearance vortex development and shock-vortex-interaction in a transonic axial compressor rotor. AIAA.

Schlechtriem S and Lotzerich M. (1997). Breakdown of tip leaksge vortices in compressors at flow conditions close to stall. ASME.

Shi K and Fu S.(2013). Study of shock/ blade tip leakage vortex/boundary layer interaction in a transonic rotor with IDDES model. ASME.

Brandstetter C and Schiffer H P.(2018).PIV measurements of the transient flow structure in the tip region of a transonic compressor near stability limit. Journal of the global power and propulsion society.

Spalart,P.R. and Allmaras,S.R. (1994).A one equation turbulence model for aerodynamic flows. Rech. Aerosp. pp, 5-21. 